\title{
Cardiovascular Risk in Young Workers with Left Bundle Branch Block
}

\author{
Monica Lamberti ${ }^{*}$, Gennaro Ratti ${ }^{2}$, Giuseppina Di Miscio ${ }^{3}$, Emilio Franciolini ${ }^{3}$, \\ Michele Pincone $^{3}$, Cristina Capogrosso ${ }^{2}$, Carlo Tedeschi ${ }^{4}$, Roberto De Rosa ${ }^{3}$ \\ ${ }^{1}$ Section of Occupational Medicine, Department of Experimental Medicine Second University of Naples, Naples, \\ Italy \\ ${ }^{2}$ Cardiology Unit, "S. Giovanni Bosco" Hospital, Naples, Italy \\ ${ }^{3}$ Cardiology and Radiology Unit, "Cliniche Riunite" Hospital, Foggia, Italy \\ ${ }^{4}$ Cardiology and Radiology Unit, "S. Gennaro" Hospital, Naples, Italy \\ Email: " monicalamberti@libero.it
}

Received 12 February 2014; revised 11 April 2014; accepted 7 May 2014

Copyright (C) 2014 by authors and Scientific Research Publishing Inc.

This work is licensed under the Creative Commons Attribution International License (CC BY).

http://creativecommons.org/licenses/by/4.0/

(c) (i) Open Access

\begin{abstract}
Aims: Left bundle branch block (LBBB) is generally associated with a poorer prognosis in comparison to normal intraventricular conduction, but also in comparison to right bundle branch block which is generally considered to be benign in the absence of an underlying cardiac disorder like congenital heart disease. In this paper we evaluate the presence of possible cardiovascular pathology in a group of nurses with a low level of cardiovascular risk factors and left bundle branch block (LBBB). Methods: During the period 2009-2013, 356 nurses (mean age: $32.6 \pm 11 \mathrm{yr}$ ) were admitted to the department of Occupational Medicine of Second University of Naples. Of these, 13 had LBBB. The evaluation of these patients has included an electrocardiogram (ECG), echocardiography, 24-h ambulatory Holter monitoring (ECG Holter), and exercise testing. Subsequently, in patients with LBBB, multislice computed coronary angiography (MSCT) has been considered. Results and Conclusion: Only in one patient we found a significant stenosis in the middle tract of left anterior descending artery. Coronary artery disease remains difficult to diagnose in some ECG findings such as acquired LBBB. For this reason, a preventive diagnose with newly developed diagnostic methods such as the multislice computed coronary angiography (MSCT) must try to account by the clinician in order to ruling out coronary artery disease (CAD) in workers with LBBB and low cardiovascular risk.
\end{abstract}

\section{Keywords}

Left Bundle Branch Block, Coronary Artery Disease, Nurses

\footnotetext{
"Corresponding author.
} 


\section{Introduction}

For its broad use as a screening tool, the electrocardiogram (ECG) has largely become one of the most common diagnostic tests performed in routine clinical practice. The finding of left bundle-branch block (LBBB) in the absence of a well-defined clinical setting raises questions and often concerns in stratifying risk of cardiovascular events [1]. LBBB may reveal an underlying coronary artery disease (CAD). In occupational medicine, the doubts become greater for judgment of work fitness; in fact an underlying CAD can be triggered by manual job. Assessment of work fitness must take into account clinical findings, which may at times be cloudy, when they indicate disease. Therefore, an extensive cardiology evaluation is mandatory among workers [2]. Cardiologists are routinely called upon to make a judgment about cardiovascular work fitness of their patients. Despite continuing technical innovations, coronary angiography remains an invasive examination and more studies report the accuracy of multislice computed coronary angiography (MSCT) to diagnose and estimate coronary stenosis in comparison with the "gold standard" conventional angiography [3]-[5].

The goal of this study was to assess presence of possible cardiovascular pathology in a group of young nurses with a low level of cardiovascular risk factors and left bundle branch block (LBBB).

\section{Methods}

Three hundred and fifty-six nurses (mean age: $32.6 \pm 11$ years), were screened between 2009 and 2013 at the Department of Occupational Medicine of the Second University of Naples. The 10-year's risk of fatal cardiovascular disease (SCORE 2003 project chart for populations at low cardiovascular risk) was low (1\%) for all patients [6]. These subjects had no previous medical history, were asymptomatic, and had normal physical examinations. Their characteristics are described in Table 1 . They underwent a full cardiology examination including resting electrocardiogram (ECG), echocardiography, 24-h ambulatory Holter monitoring (ECG Holter), and exercise testing and subsequently, in those with LBBB (thirteen had a LBBB at ECG), MSCT.

All patients gave written informed consent to the study protocol, which was approved by the local ethics committee.

LBBB was recorded on a 12-lead ECG and defined using the criteria set by the Criteria Committee of the New York Heart Association [7]: QRS interval >120 ms; slurred/notched wide and predominant R waves in leads DI, aVL, V5, and V6; slurred/notched and broad S waves in V1 and V2 with absent or small R waves; midconduction delay defined as notching or a plateau in the mid-QRS wave; ventricular activation time $50 \mathrm{~ms}$ at the onset of the QRS interval; M-shaped QRS variants with occasionally wide R waves in V5 and V6; and no initial $\mathrm{Q}$ wave over the left precordium and absence of pre-excitation.

The echocardiogram was performed using a Logic (GE Group) echocardiograph and conventional measurement (American Society of Echocardiography).

All patients underwent symptom-limited stress test according to the Bruce protocol with cycle-ergometer. All the medications patients were receiving were continued through the testing. Patient's symptoms, peak heart rate, blood pressure, and any ECG changes were noted. The exercise test was considered positive if there was $>1$-mm ST horizontal segment depression or 1-mm ST depression at $80 \mathrm{~ms}$ after the J point on the exercise ECG (GE Medical Systems).

24-h ambulatory Holter monitoring was recorded using the Holter recorders DMS 300-7 (DMS producer, Nevada, USA) with 7 leads (3 Channels) and analyzed with DM software cardioscan II. These examinations were supervised by appropriately trained cardiologists.

Table 1. Cardiovascular disease risk factors present in 13 nurses with left bundle branch block.

\begin{tabular}{cc}
\hline \multicolumn{2}{c}{ Characteristics of 13 the nurses with LBBB } \\
\hline Diabetes & $0 / 13$ \\
Smoke & $3 / 13(23 \%)$ \\
High blood pressure & $0 / 13$ \\
Family history of cardiovascular disease & $0 / 13$ \\
Dyslipidemia & $1 / 13(8 \%)$ \\
\hline
\end{tabular}


All MSCT examinations were performed with a 64-detector-row scanner (Aquilion 64 CFX; Toshiba Medical System, Tokyo, Japan) with low dose prospective acquisition. Patients with heart rate above 60 bpm received 50 $\mathrm{mg}$ atenolol orally for 3 days before scanning, or they increased their usual therapy with beta-blockers, in order to obtain a prescan heart rate of $60 \mathrm{bpm}$. The coronary artery tree was divided into 16 segments based on a modified AHA classification [8].

Coronary atherosclerosis was defined as the presence of any atherosclerotic plaque, intended as a structure $>1$ mm within or adjacent to the coronary artery lumen, which can be clearly distinguished from the vessel lumen and the surrounding pericardial tissue. A characterization of plaques, classifying them in calcified (or mostly calcified), mixed and non calcified (or mostly non calcified), is included.

Finally, it was determined whether the lesion was obstructive or not using a threshold of $50 \%$ luminal narrowing.

\section{Results}

Of 356 evaluated subjects, 13 had LBBB. All patients with LBBB were healthy, physically satisfactory, and had a low level of cardiovascular risk. They had no significant past medical or family history. They were all asymptomatic and denied any symptoms related to this conduction anomaly (chest pain, palpitations, exertional dyspnoea, presyncope). No infectious disease occurred during the past months preceding the discovery of LBBB. Physical examination was normal for all patients. ECG, Holter, and exercise testing showed permanent LBBB patterns without any other significant conduction disorders. All the patients were asymptomatic during the exercise testing. Echocardiography didn't show relevant alteration. It was decided not to perform a stress ultrasound because the acoustic window in the majority of patients appeared inadequate. MSCT was performed without complications in all patients with LBBB. Additional treatment with beta-blocker therapy was needed in 7 patients before scanning. Only in one patient MSCT found a significant stenosis in the middle tract of the left anterior descending artery (LAD) due to a non calcified plaque (Figure 1 and Figure 2). In two other patients computed angiography identified aterosclerotic non calcified and mixed plaques, without significant stenosis (<50\%) in the left circumflex artery (CX) and proximal/middle right coronary artery (RCA).

\section{Discussion}

The theoretical basis of medico-legal terminology for occupational fitness is founded on the concepts of suitability, fitness, capacity, ability and specific potential that the worker may attain during accomplishment of the job. [9] To assess fitness for a specific job, the occupational physician must consider whether the worker possesses the psycho-physical requisites normally needed to perform the job, not the top levels of such requisites [10]. Assessment of work fitness must take into account clinical and/or laboratory findings, which may at times not be clear, when these indicate "heart disease", for example, LBBB increases risk of cardiac mortality, and the prognosis is primarily determined by the underlying CAD. In many cases, particularly in adults, new onset LBBB reflects advanced and advancing heart disease. This can be illustrated by a report from the prospective Framingham Heart Study [11] [12]. In general population, most of the studies have been conducted using an

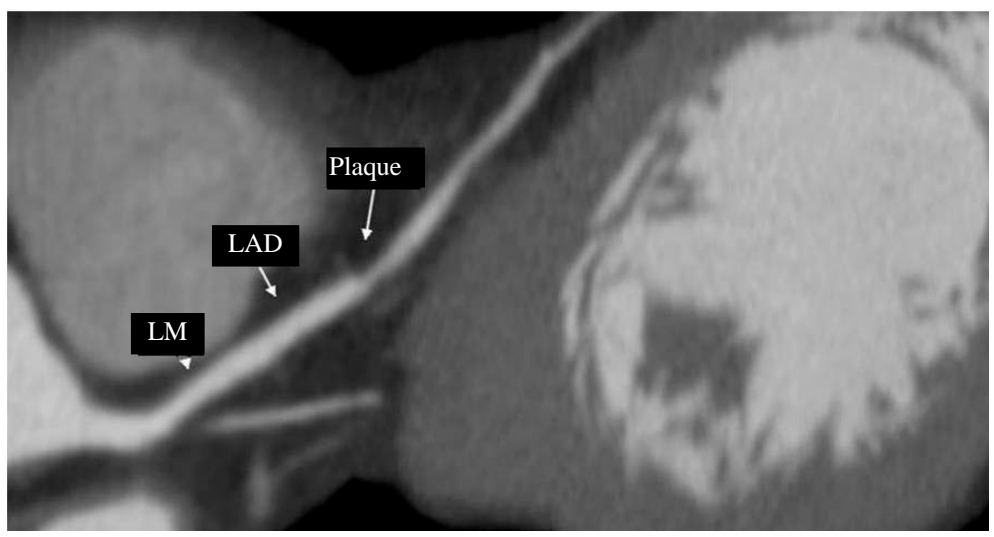

Figure 1. MPR MSCT reconstruction of noncalcified plaque on LAD. 


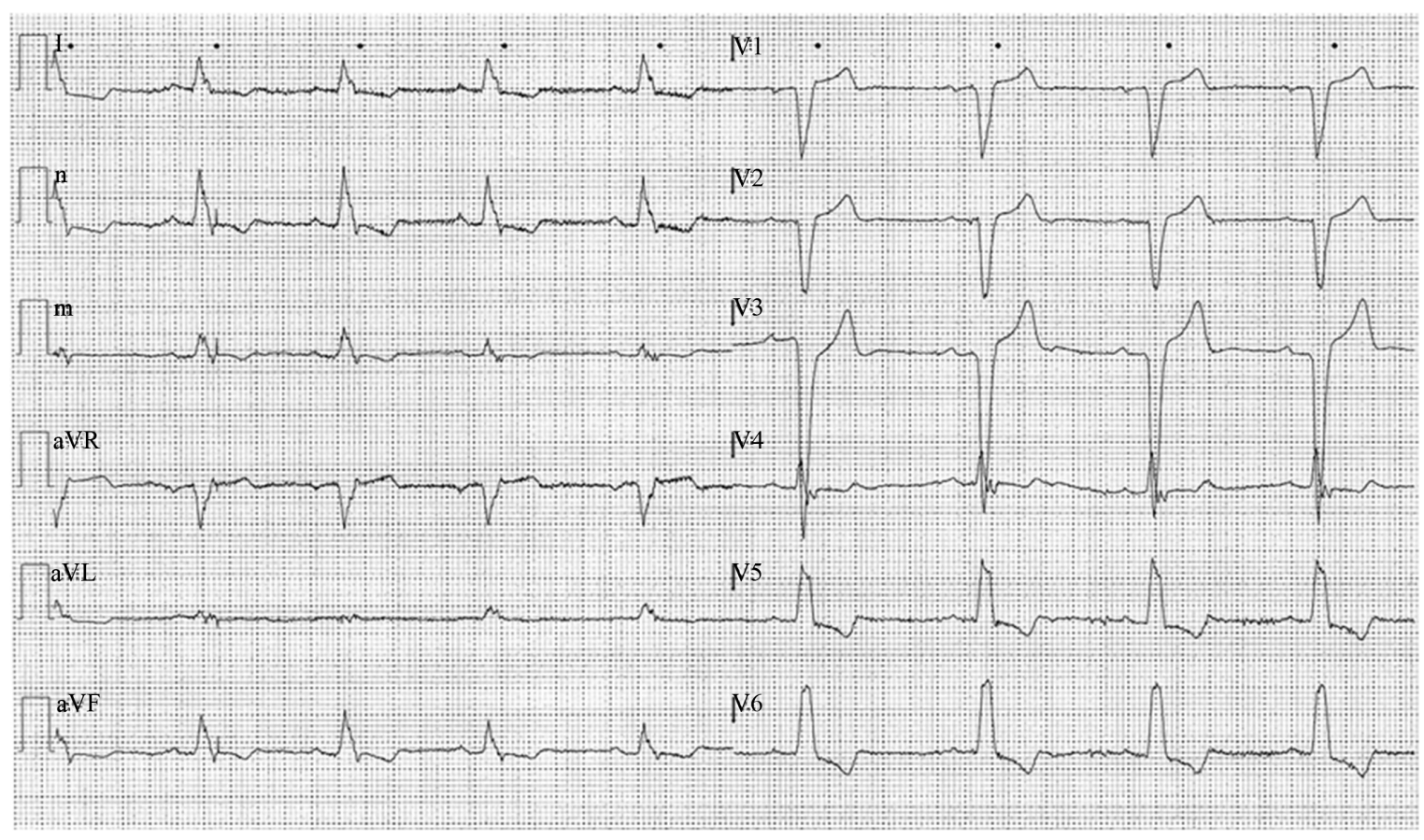

Figure 2. Electrocardiogram (ECG) of nurse with noncalcified plaque on LAD.

older, less healthy, hospital based population with, in most cases, an underlying cardiovascular disease [13]-[17]. We identified only one significant CAD in LAD and 2 non significant atherosclerotic plaques in other 2 patients in our study. Although the prognosis of LBBB in apparently healthy young subjects appears to be better, being essentially dependent on the cause of the LBBB, this study shows that in our population at low cardiovascular risk with a LBBB, CAD can be found. This could justify the new trend of preventive medicine to use noninvasive methods such as MSCT to formulate a correct diagnosis in these patients [18] [19].

The occupational physician should take into account that, unfortunately, factors such as age, and the low cardiovascular risk are not always certain criteria for exclusion of heart disease, especially when there are disorders of heart rhythm that can mask possible coronary artery disease.

For this reason, a preventive diagnose with newly developed diagnostic methods such as the MSCT must try to account by the clinician in order to ruling out CAD in manual laborers with LBBB and low cardiovascular risk.

\section{References}

[1] Francia, P., Balla, C., Paneni, F. and Volpe, M. (2007) Left Bundle-Branch Block-Pathophysiology, Prognosis, and Clinical Management. Clinical Cardiology, 30, 110-115. http://dx.doi.org/10.1002/clc.20034

[2] Picciotto, D., Verso, M.G., Grant, M. and Cannizzaro, E. (1998) Rhythm and Conduction Disorders in Formulating a Judgment of Work Fitness. Med Lav Medicina del Lavoro, 89, 387-392.

[3] Gilard, M., Le Gal, G., Cornily, J.C., Vinsonneau, U., Joret, C., Pennec, P.Y., Mansourati, J. and Boschat, J. (2007) Mid Term Prognosis of Patients with Suspected Coronary Artery Disease and Normal Multislice Computed Tomographic Findings. A Prospective Management Outcome Study. Archives of Internal Medicine, 165, 1686-1689. http://dx.doi.org/10.1001/archinte.167.15.1686

[4] Carrigan, T.P., Nair, D., Schoenhagen, P., Curtin, R.J., Popovic, Z.B., Halliburton, S., Kuzmiak, S., White, R.D., Flamm, S.D. and Desai, M.Y. (2009) Prognostic Utility of 64-Slice Computer Tomography in Patients with Suspected but No Documented Coronary Artery Disease. European Heart Journal, 30, 362-371. http://dx.doi.org/10.1093/eurheartj/ehn605

[5] Achenbach, S., Ropers, D., Hoffmann, U., et al. (2004) Assessment of Coronary Remodeling in Stenotic and Nonstenotic Coronary Atherosclerotic Lesions by Multidetector Spiral Computed Tomography. Journal of the American College of Cardiology, 43, 842-847. http://dx.doi.org/10.1016/j.jacc.2003.09.053

[6] Conroy, R.M., Pyorala, K., Fitzgerald, A.P., et al. (2003) Estimation of Ten-Risk of Cardiovascular Disease in Europe: 
The SCORE Project. European Heart Journal, 24, 987-1003. http://dx.doi.org/10.1016/S0195-668X(03)00114-3

[7] Criteria Committee of the New York Heart Association (1994) Nomenclature and Criteria for Diagnosis of Diseases of the Heart and Great Vessels. 9th Edition, Little Brown, Boston, 210-219.

[8] Austen, W.G., Edwards, J.E., Frye, R.L., et al. (1975) A Reporting System on Patients Evaluated for Coronary Artery Disease. Report of the Ad Hoc Committee for Grading of Coronary Artery Disease, Council on Cardiovascular Surgery, American Heart Association. Circulation, 51, 5-40. http://dx.doi.org/10.1161/01.CIR.51.4.5

[9] Zanettini, R. and Farina, G. (2004) Work Capacity and Re-Entry into the Workforce for the Cardiac Patient. Med Lav Medicina del Lavoro, 95, 140-144.

[10] Lee, S.M. and Koh, D. (2008) Fitness to Work: Legal Pitfalls. Annals of the Academy of Medicine, Singapore, 37, 236240.

[11] Schneider, J.F., Thomas Jr., H.E., Kreger, B.E., et al. (1979) Newly Acquired Left Bundle Branch Block. The Framingham Study. Annals of Internal Medicine, 90, 303-310. http://dx.doi.org/10.7326/0003-4819-90-3-303

[12] Hesse, B., Diaz, L.A., Snader, C.E., Blackstone, E.H. and Lauer, M.S. (2001) Complete Bundle Branch Block as an Independent Predictor of All-Cause Mortality: Report of 7,073 Patients Referred for Nuclear Exercise Testing. American Journal of Medicine, 110, 253-259. http://dx.doi.org/10.1016/S0002-9343(00)00713-0

[13] Eriksson, P., Wilhelmsen, L. and Rosengren, A. (2005) Bundle-Branch Block in Middleaged Men: Risk of Complications and Death over 28 Years. The Primary Prevention Study in Goteborg, Sweden. European Heart Journal, 26, 2300-2306. http://dx.doi.org/10.1093/eurheartj/ehi580

[14] Eriksson, P., Hanson, P.O., Eriksson, H., et al. (1998) Bundle Branch Block in a General Population. Circulation, 98, 2494-2500. http://dx.doi.org/10.1161/01.CIR.98.22.2494

[15] Fahy, J.G., Pinski, L.S., Miller, P.D., et al. (1996) Natural History of Isolated Bundle Branch Block. American Journal of Cardiology, 77, 1185-1190. http://dx.doi.org/10.1016/S0002-9149(96)00160-9

[16] Hardason, T., Arnason, A., Eliasson, G.J., et al. (1987) Left Bundle Branch Block: Prevalence, Incidence, Follow-Up and Outcome. European Heart Journal, 8, 1075-1079.

[17] Kreger, B.E., Anderson, K.M. and Kannel, W.B. (1989) Prevalence of Intraventricular Block in the General Population: The Framingham Study. American Heart Journal, 117, 903-910. http://dx.doi.org/10.1016/0002-8703(89)90630-3

[18] Haberl, R., Tittus, J., Bohme, E., et al. (2005) Multislice Spiral Computed Tomographic Angiography of Coronary Arteries in Patients with Suspected Coronary Artery Disease: An Effective Filter before Catheter Angiography? American Heart Journal, 149, 1112-1119. http://dx.doi.org/10.1016/j.ahj.2005.02.048

[19] Goldstein, J.A., Gallagher, M.J., O’Neill, W.W., et al. (2007) A Randomized Controlled Trial of Multi-Slice Coronary Computed Tomography for Evaluation of Acute Chest Pain. Journal of the American College of Cardiology, 49, 863871. http://dx.doi.org/10.1016/j.jacc.2006.08.064 